\title{
VIII.
}

\section{Ueber das Verhältnis von Staat und Schule in der römischen Kaiserzeit.}

Eine systematische Einwirkung seǐens der Staatsautorität auf das geistige Leken der im römischen Reiche vereinigten Völker, insbesondere der herrschenden Römer selbst, dann auch der Kulturträger des Reichs, der Hellenen, ist erst mit der Gründung der Monarchie wahrzunehmen. Die Herrschaft eines einzigen widerspricht den bisherigen Anschauungen von der Freiheit des römischen Volkes. Um nun einen Wandel in den eingewurzelten Ansichten hervorzubringen, benützt Augustus in seiner klugen Art auch die Literatur. Er und seine Freunde wenden ihre Gunst den sich emporringenden Talenten zu, ein Mäcenas und Messala vielleicht aus wirklichem Interesse, nicht etwa aus Langeweile infolge der unmöglich gewordenen politischen Tätigkeit, die den Cicero zum Philosophen, den Asinius Pollio zum Kunstrichter gemacht hatte, der Princeps selbst vor allem aus politischer Berechnung. Die Tendenz der Aeneis wie der Römeroden des Horaz ist es, die neue Dynastie durch poetische Verherrlichung populär zu machen, die gens Julia als das von den Göttern gewollte und begünstigte nationale Herrschergeschlecht den z. T. noch unversöhnten Zeitgenossen zu empfehlen. Nicht in gleichem Grade scheint sich der durchaus römisch fühlende Sieger von Aktium um panegyrische Leistungen griechischer Dichter bemüht zu haben, wenn auch die Epigramme des Krinagoras Beifall bei Hofe fanden. Aber mit dem Erstarken des hellenischen Selbstbewußtseins und der Renaissance der griechischen Literatur, mit dem Aufblühen der in der gebildeten Welt tonangebenden Sophistik. ward die Aufmerksamkeit der 
Herrscher wiederum auf das erste Kulturvolk des Reiches gelenkt, dessen Bedeutung für den Bestand desselben im Kampf mit dem Orient immer klarer zutage trat. Beachtung forderte andrerseits der Bund der Stoa und des Kynismus mit den antimonarchischen römischen Elementen.

Der Philhellenismus eines Hadrian, der sich auch in der Auszeichnung griechischer Gelehrter kundgab ${ }^{1}$ ), war nur Mittel zum Zwecke der Stärkung des Reichsgedankens. Trug doch dieser scheinbare Freund der Literatur seine Verachtung gelehrten Wesens jedenfalls in seinen Kreisen in echt römischer Weise drastisch genug zur Schau ${ }^{2}$ ), so daß wir unwillkürlich an Friedrich Wilhelm I. und sein Tabakskollegium erinnert werden.

Der Panegyrikus auf die Monarchie, den des Aelius Aristides Rede auf Rom darstellt ${ }^{3}$ ), ist der Dank für die Gunst, welche Antoninus Pius den griechischen Sophisten erweist, ebenso ist der Ausfall des Appian ${ }^{4}$ ) gegen die Kyniker, die Hungerleider, die den Herrschern Opposition machten, eine Kundgebung der loyalen Gesinnung des alexandrinischen Literaten, der es bis zum kaiserlichen Prokurator brachte. Wie einflußreiche Priester- und Adelsfamilien in den Städten des Ostens durch Verleihung des römischen Bürgerrechts, der Würde des Kaiserpriestertums und sonstige Auszeichnungen, durch Aufnahme von Familienmitgliedern in das römische Offizierskorps und den Reichsbeamtenstand für Rom gewonnen wurden, so auch die griechischen Schriftsteller, Gelehrten, Aerzte usw., die Verbindung mit Rom und dem Kaisertum suchten. Es sei nur an Flavius Josephus, Flavius Arrianus, Cassius Dio Cocceianus, Aelius Aristides, Flavius Philostratus, Aelius Herodianus, Claudius Galenus und vor allem an Lucian erinnert, der noch in seinem Alter um die Aussicht auf eine

1) Ludwig Hahn, Das Kaisertum, Leipz. 1913, $14 \mathrm{f}$.

2) Vita Hadr. 15, 10: professores om nium artium semper ut doctior risit, contempsit, obtrivit; cf. c. 16 und vita Helii 4, 2. - Vgl. auch F. Gregorovius, Gesch. d. röm. Kaisers Hadrian und seiner Zeit ${ }^{3} 347$.

8) Vgl. L. Hahn a. 0. a. O. $24 \mathrm{ffi}$.

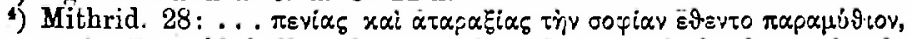

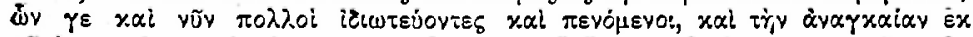

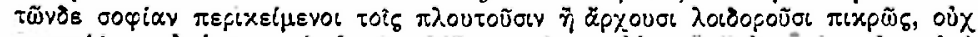

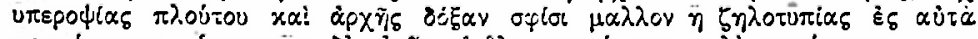

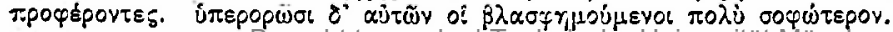

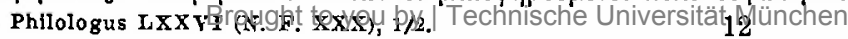


kaiserliche Prokuratur vou national hellenischen ins römische Lager übergeht.

Bei der großen Bedeutung, die kluge Herrscher der Förderung der romfreundlichen und monarchischen Strömung in der Gedankenwelt ihrer Zeitgenossen beimaken, ist es natürlich, daß die Staatsleitung auch Einfluf auf die kommenden Geschlechter vermittelst der Einwirkung auf die von den regierenden Gewalten lange übersehenen, privater ${ }^{5}$ ) Fürsorge überlassenen Schulen gewinuen wollte, zumal auf die höheren Schulen, an welchen die Söhne aus den oberen Schichten, die zukünftigen Offiziere und Beamten ihre Ausbildung bekamen und sich ihre Lebensanschaungen auf Grund der von ihren Lehrern vorgetragenen Ideen bildeten.

Der Kampf der republikanischen Opposition gegen die monarchische Staatsform ist zugleich ein Kampf um die Gewinnung der Jugend, ein Kampf um die Schule, d. h. um die in der Jugendbildung zu vertretenden Grundsätze. Thrasea Pätus, der Freund und Verwandte des Stoikers A. Persius Flaccus, stand unter dem Einflusse des Kynikers Demetrios von Sunion ${ }^{6}$ ), der auch von Seneka hoch verehrt wurde ${ }^{7}$ ). Wenn der sehr duldsame Vespasian ${ }^{8}$ ) sich gezwungen sah über Demetrios die Strafe der Verbannung, über Helvidius Priscus, den Schwiegersohn des Pätus Thrasea, die Todesstrafe zu verhängen, so tat er dies im Interesse des Staates, da er den hartnäckigen, in seinen Augen unvernünftigen Widerstand, den eine Schule von Feinden der Monarchie der konsolidierten Verfassung des Reiches leistete, als der für das Wohl des

5) Auf den Mangel an Fürsorge für die Erziehung des heranwachsenden Geschlechts, der dem römischen Regierungssystem eigen sei, deutet eine merkwürdige seußerung in einem angeblichen Briefe de Apollonius von Tyana hin, Epist. 54 I p. 358 Kays.: 'A

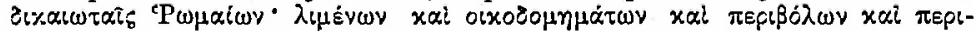

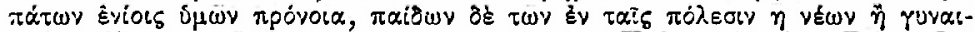

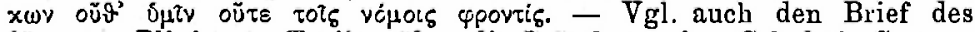
jüngeren Plinius an Tacitus über die Gründung einer Schule in Comum (ep. IV 13). - Bei der Unterstützung der alimentarii pueri et puellae (seit Nerva) kommt vor allem Italien in Betracht. Dieselbe galt auch der leiłlichen, nicht der geistigen Erziehung. Vgl. Kubitschels s. v. alimenta bei Pauly-Wissowa.

-) Tac. ann. XVI 34.

3) De benef. VII 1,3 u. $8,2$.

8) Suet. Vespas. 13. - Dio 6. 13. 
Reiches verantwortliche Faktor nicht weiter dulden konnte. Wird ja unnützer Radikalismus auch von Tacitus verurteilt. Die Vertreibung der oppositionellen Philosophen aus Rom unter Vespasian und Domitian stellt wie in der republikanischen Zeit die Ausweisung der Philosophen in àen J. 173 und 161, wie die schnelle Abfertigung der drei athenischen Gesandten (155), von denen Cato $^{9}$ ) meinte, sie sollten so bald als möglich in thre Schulen zurückkehren und dort die jungen Leute ihres Landes unterrichten, den Söhnen Roms aber möge die Achtung vor Gesetz und Obrigkeit erhalten bleiben, wie das Verbot rom Senate nicht genehmigter Kulte nur einen Akt der Selbstwehr des nur durch die Monarchie existenzfähigen Staates dar. In gleicher Absicht schreitet der heidnische Staat im Interesse der anerkannten Staatsreligion und des Kaiserkults gegen das Christentum ein.

Die unter den Flavischen Kaisern wieder dreister auftretende und sich forterbende republikanische Opposition mußte den Gedanken nahe bringen, daß dem Staate ein Oberaufsichtsrecht über die Schule und ihre Lehrer in dem Sinne zustehe, dak in der Jugend keine Ideen verbreitet werden dürften, die eine Gefahr für den Bestand des monarchischen Regiments bedeuten konnten. Einen Philosophen wie Peregrinus Proteus, der sich in Schmähreden gegen den Kaiser und die römische Herrschaft ergehe, könne man, meint auch später noch ein Stadtpräfekt, in der Stadt Rom nicht brauchen ${ }^{10}$ ).

Wenn nun Vespasian ${ }^{11}$ ) für die lateinischen und griechischen Thetoren in Rom d. h. also für diejenigen Lehrer, welche vorzugsweise Jünglinge aus den höheren Ständen unterrichten und auf deren geistige Entwicklung im empfänglichsten Alter einwirken, ihren Lebensanschauungen eine bestimmte Richtung geben und ähnlich wie die Lehrer unserer Universitäten den Geist der Zeit und ihre Literatur mitbestimmen konnten, einen Gehalt von 100000 Sesterzen festsetzte, so ward er bei seiner bekannten Sparsankeit jedenfalls durch besondere Gründe der Staatsraison zu diesem Schritt bewogen. Die Autorität des

9) Plut. Cato mai. c. 22 f.

${ }^{10)}$ Vgl. Jakob Bernays, Lucian und die Kyniker, Berl. 1879, $7 \mathrm{f}$.

11) Suet. Vespas. c. 18: primus e fisco Latinis Graecisque rhetoribus annua centena constituit. - O. Hirschfeld, Berl. Sitzungsber. 1905, 916. 
Kaisertums sollte auch durch die Schule gestützt werden. Zur Besoldung fügte Vespasian ${ }^{12}$ ) noch Privilegien für die magistri grammatici et oratores sive sophistae et philosophi wie die Befreiung von der Last der Einquartierung. Unter den philosophi verstand der Kaiser jedenfalls nicht die Kyniker, sondern die Lehrer der gewissermaßen staatlich anerkannten Philosophenschulen, deren 4 Scholarchen in Athen wie auch der

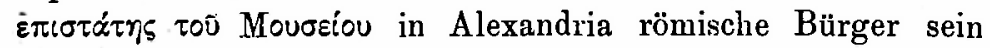
mußten ${ }^{13}$ ), eine Bestimmung, die offenbar schon vor der Zeit Hadrians Geltung hatte. Hadrian schuf in Rom in dem von ihm gegründeten Athenäum eine Staatsprofessur für Rhetorik,

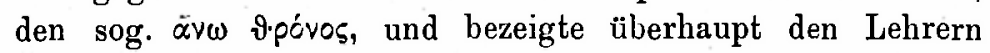
und Männern der Wissenschaft seine Gunst, indem er ihnen, wenn sie ihren Dienst nicht mehr versehen konnten, Pensionen anwies ${ }^{14}$ ). Auch Juvenal ${ }^{15}$ ) richtet seinen Blick auf den Kaiser, von dem er meint, die Hoffnung für die Studien gründe sich allein auf ihn; aber es scheint, dafi die Sonne der kaiserlichen Gunst nur über höfischen Dichtern wie Pankrates und Mesomedes aufging, die ihre knechtische Gesinnung durch Verherrlichung des kaiserlichen Lieblings Antinoos zeigten ${ }^{\mathbf{1 6}}$ ).

Diese Politik, die darauf abzielte, die öffentliche Meinung durch die damaligen Vertreter derselben, die Rhetoren, Sophisten, Philosophen, Literaten, für Rom und die Monarchie zu gewinnen, ward konsequent von Antoninus Pius, Marc-Aurel und Commodus fortgesetzt. Antoninus Pius ${ }^{17}$ ) gewährte den Rhetoren und Philosophen in allen Provinzen Ehrenbesoldungen und Privilegien. Die Städte folgten dem von oben gegebenen Beispiel.

19) Digest. L, 4, 18, 30; cf. Cod. Theod. XIII, 3, 3. 16. 17.

13) CJL. III. 12283 . $-R$. Dareste, Un rescrit de l'empereur Hadrien in Nouv, revue hist. de droit français et etranger XVI (1892) $622 \mathrm{ff}$.

14) Philostrat. vit. soph. I, 24, 3 ex; II, 10, 5. - vita Hadr. 16, 2. Aur. Vict. Caess. 14, 1. - vita Helii 4, 2.

15) VII, 1: Et spes et ratio studiorum in Caesare tantum.

16) Vgl. Gust. Fr. Hertzberg, Gesch. Griechenlands unter der Herrschaft der Römer, II., Halle $1868,348$.

ii) Vita 11, 3: rhetoribus et philosophis per omnes provincias et honores et salaria detulit. - Digest. 27, 1, 6 bringt Modestinus ein

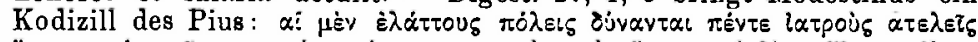

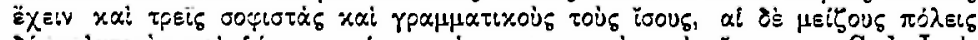

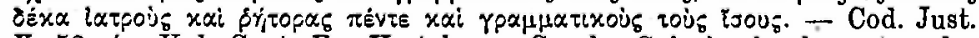
$\mathrm{X}, 53,4$. Vgl. Gust. Fr. Hertzberg, Gesch. Griechenlands unter der Herrschaft der Römer, Halle 1875, III 84 An. 44. 
Kaiser Marcus gab Lehrern aller wissenschaftlichen Fächer in Athen Gehälter und ward gewissermaßen der Gründer der Universität Athen ${ }^{18}$ ). Dabei bediente er sich auch des Rates des Herodes Atticus. Als Besoldung, welche der Kaiser den öffentlichen Lehrern aus den 4 staatlich anerkannten Philosophenschulen der Stoiker, Platoniker, Epikureer und Peripatetiker aussetzte, werden 10000 Drachmen angegeben ${ }^{19}$ ). Bei ihm, dem ideal gesinnten Herrscher, mag allerdings mehr reine Liebe zu Wissenschaft und philosophischer Denkart als die Staatsraison das Motiv gewesen sein, wenn er auch die Notwendigkeit eines Bundes der äußeren Macht des Römertums mit der geistigen des Hellenismus gegenïber den immer mehr andrängenden Kräften des Orients, zumal des Christentums gefühlt haben mag, eines Bundes, der später bes. in den Zeiten Julians, des begeisterten Verehrers und Jüngers

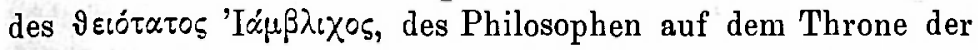
Cäsaren, als der Neuplatonismus dem umsonst mit seinen juristischen Waffen sich des neuen Geistes erwehrenden römischen Staate die letzten geistigen Kampfmittel lieferte, immer mehr in Erscheinung tritt.

Dem herkömmlichen System, daß der Kaiser durch Erteilung von Privilegien die gebildeten und loyalen Elemente für ihre gemeinnützige Tätigkeit als Philosophen, Rhetoren und Aerzte belohnt, folgt auch Commodus ${ }^{20}$ ).

Die Kaiser gewährten die Gehälter und Benefizien natürlich nur solchen Lehrern, die ihnen als würdig empfohlen wurden oder deren Befähigung und Gesinnung sie selbst kennen gelernt hatten ${ }^{21}$ ). Auch im Falle der Ernennung durch den Statthalter oder praefectus praetorio war natürlich wie

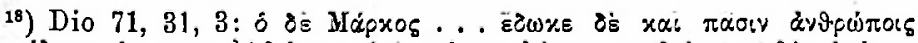

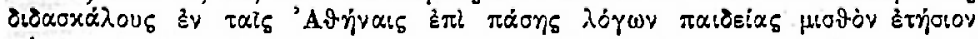

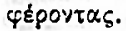
S. 490 .

19) Lucian, Eunuch. c. 3. - Diels, Arch. f. Gesch. d. Philos. 1891

20) Digest. XXVII, 1, 6, 8; cf. Br. Keil, Gött. Nachr. 1905, 415.

21) Vgl. Fertzberg a. 0. a. O. III $18 \mathrm{ff}, 104 .-$ Cod. Theod. XII, 2,1 (vom J. 349): Imp. Constantius A. ad Marcellinum comitem orientis : ... Nulli salarium tribuatur ex viribus reipublicae nisi ei, qui iubentibus nobis specialiter fuerit consecutus. XIII 3.5 (vom J. 362) und 7 (vom J. 369). 
überall die kaiserliche Genehmigung vorbehalten ${ }^{22}$ ). Die Verhältnisse mögen ähnlich gewesen sein wie bei der Besetzung erledigter Stellen des alexandrinischen Museums, die in letzter Instanz Sache des Kaisers, des Nachfolgers der ägyptischen Könige, war, wie denn z. B. der Sophist Polemon durch die Gunst Hadrians Mitglied des Museums wurde ${ }^{23}$ ). Verdienten

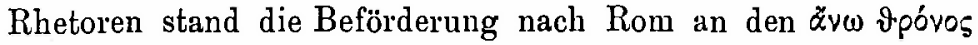
oder an die Bibliotheken der Hauptstadt oder schlieflich in die griechische Abteilung des kaiserlichen Sekretariats in Aussicht. Es winkte ihnen ferner der Ritterrang, ja die Aufnahme in den Senat ${ }^{24}$ ). So ward z. B. der Grammatiker Dionysios aus Alexandria ${ }^{25}$ ), der von Nero bis Trajan in Rom lebte, Vorstand der kaiserlichen Bibliotheken und Sekretär ab epistulis, der Rhetor und kaiserliche Sekretär. C. Avidius Heliodorus, der Vater des Prätendenten Avidius Cassius, aber brachte es bis zum Präfekten von Aegypten ${ }^{26}$ ). Der Sophist Antipater von Hierapolis, „Lehrer der Götter" d. h. Prinzenerzieher bei Septimius Severus, erhielt sogar die Stadthalterschaft von Bithynien ${ }^{27}$ ).

Die Qualität der Lehrer, die vom Staate oder den Gemeinden unterstützt wurden, war einer Prüfung oder Begutachtung unterworfen, wie Edikte des Gordian ${ }^{28}$ ), des Julian ${ }^{29}$ ), des Valentinian und Valens ${ }^{30}$ ) zeigen. Professoren und vor

22) Hertzberg a. o. a. O. III 313 ff. - Rauschen, Das griech.-röm. Schulwesen zur Zeit des ausgehenden antilen Heidentums, Bonn. $1900,21$.

$\left.{ }^{23}\right)$ Philostrat. vit. soph. I, 25, 3. - Weitere Mitglieder gibt Mommsen, R. G. V 590 an. - Vgl. auch Grasberger, Erziehung und Unterricht im klass. Altert. III 453.

24) Beispiele bei L. Friedländer, Sittengeschichte Roms I ${ }^{6}, 180 \mathrm{ff}$. Vgl. O. Hirschfeld, Die kais. Verwaltungsbeamten ${ }^{2} 318 \mathrm{ff}$. 323 A. 1.

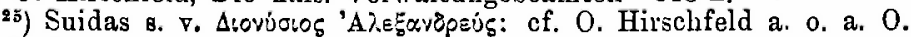

26) Friediänder a. o. a. O. I $185 \mathrm{f}$.

27) Philostrat. vit. soph. II, 24, 2.

${ }^{28}$ ) Cod. Just. X, 53, 2: Grammaticos seu oratores decreto ordinis probatos, si non se utiles studentibus praebeant, dento ab eodem ordine reprobari posse incognitum non est; cf. auch Cod. Theod. VII, 2, 1.

29) S. U. An. 41.

30) Cod. Theod. XIII 3, 7 an den praef. praet. Probus: Reddatur unusquisque patriae suaè, qui labitum philosopbiae indebite et insolenter usurpare cognoscitur, exceptis his, qui a probatissimis adprobati ab hac debent conluvione secerni. - Cf. ibid. 11: Impp. Valens, Gratianus et Valentinianus A. A. A. Antonio praef. praet. Galliarum: 
Ueber d. Verhältnis von Staat u. Schule i. d. rom. Kaiserzeit. 183

allem Aerzte konnten auch den Rang eines comes erster oder zweiter Klasse erreichen ${ }^{31}$ ). Welche Eigenschaften, Kenntnisse und Fähigkeiten man von Hochschullehrern, die einer derartigen Auszeichnung würdig befunden werden sollten, verlangte, zeigt das Edikt ${ }^{32}$ ) Theodosius' II. vom J. 425 De professoribus, qui in urbe Constantinopolitana docentes ex lege merucrint comitivam.

Die bekannten Worte, die Septimius Severus anf dem

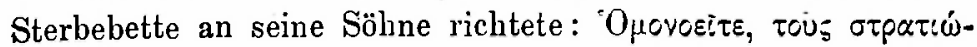

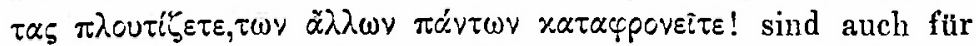
das Verhältnis von Staat und Schule in der Zeit des Soldatenkaisertums bezeichnend. Zwar erbte sich die Einrichtung der Besoldung und Privilegien für die Vertreter der Wissenschaften fort ${ }^{33}$ ), wie sich auch an der lebhaften Konkurrenz bei der Besetzung der Lehrstühle in Athen zur Zeit der Severe er-

Per omnem dioecesim commissam magnificentiae tuae frequentissimis in civitatibus ... praeceptorum optimi quique erudiendae praesideant iuventuti: rhetores loquimur et grammaticos Atticae Romanaeque doctrinac. Darauf wird die Gewährung der annonue bestimmt, darunter auch Graeco (grammatico), si qui dignus repperiri potuerit.

31) Cod. Theod. XIII 3, 17 von J. 414: Impp. Honorius et Theodosius Helioni magistro officiorum: Artium liberalium professoribus ac praecipue medicis, qui cum comitivae primi ordinis ac secundi militant dignitate, privilegia et beneficia a retro principibus praestita nec non etiam nova ipsis eor umque filiis clementia nostra detulit, ut cohaerens sanctio protestatur: quae tenaciter observari oportet; of. VI, 21, 1.

32) Cod. Theod. VI 21, 1: Imp. Theodosius A. et Valentinianus Caesar Theofilo praef. urbi: Grammaticos Graecos Helladium et Syrianum, Latinum Theofilum, sofistas Martinum et Maximum et iuris peritum Leontium placuit honorari codicillis comitivae ordinis primae jam nunc a nostra mriestate perceptis, ita ut eorum qui sunt ex vicariis dignitate potiantur. Qin in re quicumque alii ad id doctrinae genus, quod unusquisque profitetur, ordinati prodentur, si laudabilem in se probis moribus vitam esse monstraverint, si docendi peritiam facundiamque dicendi, interpretandi subtilitatem, copiam disserendi se habere patefecerint et coetu amplissimo iudicante digni fuerint aestimati, qui in memorato auditorio professorum fungantur officio, hi quoque, cum ad viginti annos observatione iugi ac sedulo docendi labore pervenerint, isdem, quibus praedicti viri, dignitatibus perfruantur. - Ueber die Universität Kpel s. L. Hahn in der Festgabe für M. v. Schanz, Würzb. 1912 , S. $180 \mathrm{ff}$.

s3) Der Jurist Herennius Modestinus unterscheidet (Digest. XXVII, 1,6)

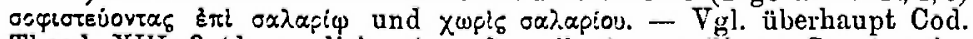
Theod. XIII, 3 (de medicis et professoribus), wo die zu Gunsten der Aerzte und Lehrer gegebenen Gesetze von Konstantin bis Theodosius II. angegeben sind. Dieselben sind meist an den praef. praet od. den praef. urbi od. den imagister officiorum gerichtet. Weitere Reskripte de professoribus et medicis gibt der Cod. Just. $X 53$. 
kennen läßt ${ }^{34}$ ), und Severus Alexander trug Sorge, daß Lehrer, Aerzte, Techniker und Architekten - aber auch - bezeichnend für den Einfluß, den man ihnen in diesen Zeiten des Verfalls antiken Denkens beimaß - Opferschauer und Astrologen Gehälter und Lehrsäle bekamen ${ }^{35}$ ), aber in den Wirren der inneren und äußeren Kriege erhob allmählich der Partikularismus sein Haupt und das Streben, durch Einwirkung auf Erziehung und Schule das Interesse für den einen römischen Kaiser und das eine römische Reich bei der so vielfach in Rasse und Sprache divergierenden Bevölkerung zu erhalten, verlor sich mit der zunehmenden Unfähigkeit des römischen wie hellenischen Elements, mit der Macht der Waffen und Gesetze einerseits und mit der der Wissenschaft andrerseits die auf die Obmacht des Geistes der beiden arischen Völker gegründete Zentralisation aufrecht zu erhalten. Jetzt zeigten sich deutlich die antiarischen Kräfte. Die Religion der unteren Schichten, der Armen und Beladenen, die mit dem Staate, solange er heidnisch blieb, nicht paktieren konnte, vielmehr die Kräfte desselben durch das passive Verhalten ihrer Bekenner lähmte ${ }^{36}$ ), war, von der Schulweisheit der Philosophen wie von der Prunkrednerei der Sophisten zumeist hochmütig übersehen oder als „barbarisch" erachtet, zu einer ungeheuren Macht, zu einem Staat im Staate geworden. Jetzt zeigt es sich, daß auch das Volk, zu dessen Befriedigung die kaiserliche Regierung panem ct circenses für gentigend gehalten, das sie den oberen Schichten gegenüber ignoriert hatte $^{37}$ ), zu eigenem Denken erwacht war und beseligt von der befreienden Lehre des Erlösers vom Heil der Welt die ganze Bildung der Zeit, die im Vergleich mit dem Evangelium als inhaltsleer und nichtig, ja sogar, weil mit der Sünde des Heidentums befleckt, als verabscheuenswert erschien, mit einem den Heiden unbegreiflichen Fanatismus zurückwies.

Als das Ziel, das sich einst schon Augustus gesetzt und

3i) Philostrat. vit. soph. II c. $26 \mathrm{f}$.

35) Vita Alex. Sev. c. 41, 4: Rhetoribus, grammaticis, medicis haruspicibus, mathematicis, mechanicis, architectis salaria instituit et auditoria decrevit.

$\left.{ }^{86}\right)$ Tertull. Apolog. c. 38. - Minuc. Fel. Octavius c. 8 ex.

s7) Cf, den Brief des Apollonius von Tyana o. An. 5. 
die folgenden Cäsaren stets im Auge behalten hatten, die Beseitigung der philosophischen und literarischen Opposition zu einem guten Teile erreicht war, als selbst ein Lucian zur nicht geringen Verwunderung seiner einem hellenischen Nationalismus huldigenden Freunde um den Preis eines einträglichen Amtes seinen Frieden mit Rom geschlossen hatte, als die Abhängigkeit der Vertreter der griechischen Literatur, der sophistischen Prunk- und Festredner wie der die ausgetretenen Geleise verfolgenden Schulphilosophen von der Gunst der römischen Kaiser offen zutage trat ${ }^{38}$ ), da hatte schon das vom Hellenisnus wie von Rom hochgehaltene und gestützte Bildungsideal der Spätantike für einen großen Teil der Bevölkerung des Reiches jeden Wert verloren und weder der von

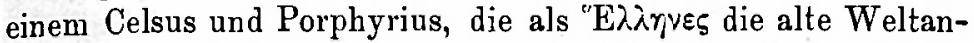
schauung vertraten, geführte geistige Kampf noch die Edikte und Gewaltmaßregeln Roms zur Zeit eines Decius und Diokletian vormochten es, den neuen Geist zu bannen und das siegreiche Vordringen der dem heidnischen Staate wie der heidnischen Bildung Verderben drohenden Lehre zu verhindern. Die Staatsleiter erkannten .jetzt auch die Wichtigkeit der Schule für den großen Kulturkampf zwischen der antiken und christlichen Weltanschauung. Schon im Anfange des 3. Jhs. war die alexandrinische Katechetenschule aufgelöst worden ${ }^{\mathbf{3 9}}$ ).

Der Christenverfolger Maximinus griff dabei zu einer merkwürdigen Maßregel, welche uns zeigt, daß man schon damals von der Richtigkeit des Satzes überzeugt war, daß dem die Zukunft gehöre, der die Schule benerrsche. Er ließ̧ nämlich ${ }^{40}$ ) die unterschobenen Acta Pilati et Christi, welche von

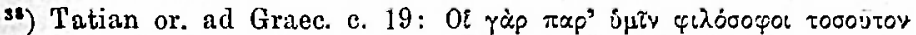

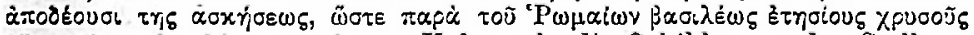

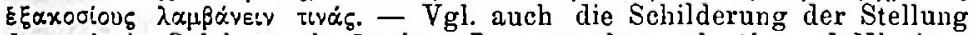
der griech. Gelehrten in Lucians De mercede conductis und Nigrinus und dazu den Giebener Papyrus I, Nr. 80 (E. Kornemann und Paul M. Meyer, Griech. Papyri zu Gieß3en, Leipz. 1913), nach dem die Hauslehrer wie Domestiken behandelt worden zu sein scheinen.

39) J. E. Weis, Christenverfolgungen, München 1899, $132 \mathrm{ff}$.

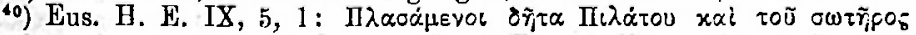

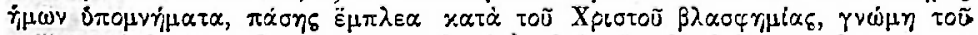

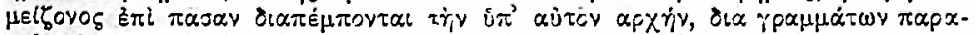

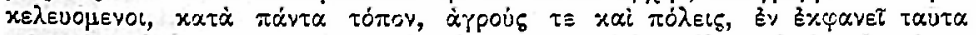

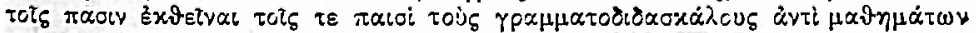

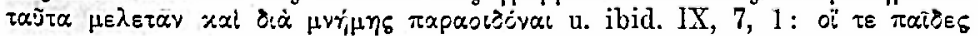


Schmähungen gegen den Erlöser strotzten, nicht nur allenthalben verbreiten und öffentlich bekannt geben, sondern verordnete auch, daf die Lehrer sie ihre Schulkinder auswendig lernen lassen sollten, so dak dieselben die Beschimpfungen dieser Akten täglich im Munde führten. Die Schule wird also in gröbster Art als Kampfmittel benützt und offenbar als dem Staate durchaus fügsam und dienstbar betrachtet und behandelt. Es müssen also wohl die Statthalter und deren Unterbeamte die Befugnis besessen haben, die Unterrichtstätigkeit auch in den Elementarschulen durch Verordnungen in gewissen Fällen zu bestimmen und zu kontrollieren.

In weit feinerer Weise suchte bekanntlich Julian die Schule gegen das siegreiche Christentum auszuspielen. In seinem „Rhetorenedikt" verbot er, dafi christliche Lehrer Unterricht in den, hellenischen $d$. h. heidnischen Wissenschaften gäben ${ }^{41}$ ). Nach einem 362 erlassenen Gesetz des Kaisers erlangten die Professoren die Erlaubnis zur Abhaltung von Vorträgen nur, wenn sie nach dem Gutachten der Dekurionen dafür qualifiziert waren: zudem behielt sich der Kaiser noch eigens die Bestätigung vor ${ }^{\mathbf{4 2}}$ ). Damit waren die Schulen, insbesondere die Hochschulen, unter die strengste staatliche Kontrolle gestellt, es war ilmen vorgeschrieben, in welchem Geiste der Unterricht zu erteilen sei ${ }^{43}$ ).

Aber wenn Julian hoffte, das Christentum durch solche Makregeln zu einer barbarischen Religion für Ungebildete zu

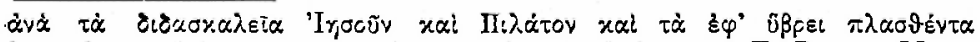

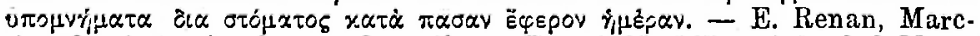
Aurele et la fin du monde antique, Par. 1882, 345 meint, daß MarcA urel mittelst der Schule dem Christentum mehr Abbruch getan hătte als durch Verfolgung und gerichtliche Bestrafung der christen.

$\left.{ }^{11}\right)$ Amm. Marcellin. XXV 4, 20: Inter quae erat illud inclemens ..., quod docere vetuit magistros riletoricos et grammaticos christianos, ni transissent ad numinum cultum. - Jon. Chrys. hom. in Juvent. et

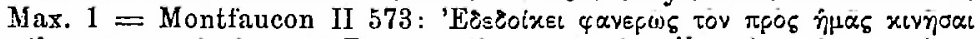

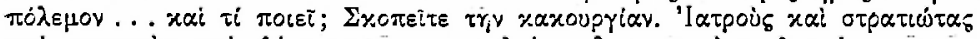

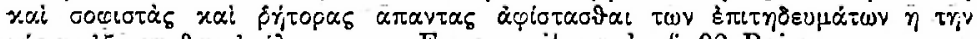

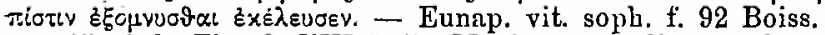

${ }^{42}$ ) Cod. Theod. XIII 3, 5: Magistros studiorum doctoresque excellere oportet moribus primum, deinde facundia. Sed quia singulis civitatibus adesse ipse non possum, iubeo, quisque docere vult, non repente nec temere prosiliat ad hoc munus, sed iudicio ordinis probatus decretum curialium mereatur optimorum conspirante consensu. Hoc enim decretum ad me tractandum referetur, ut altiore quodam honore nostro iudicio studiis civitatum accedant; cf. ibid, 6 .

43) Vgl. auch Albert Müller im Philologus Bd. 69 (1910) 292 ff. 
stempeln ${ }^{44}$ ), durch eine Renaissance des Heidentums in Staatskirche und Schule den neuen Ideen den Boden zu entziehen, so täuschte er sich. Die altersschwach gewordene heidnische Bildung vermochte den ungleichen Kampf nicht zu bestehen, sie war dem Untergang geweiht ${ }^{45}$ ), wenn sie sich nicht dem christlichen Bildungsideale als Dienerin aubequemte.

Aber auch die christlichen Kaiser fühlten die Oberaufsicht über die Schule als weiter notwendig. Nachdem das Christentum zur Stantsreligion geworden war, lag es natürlich im Interesse der jeweils staatlich anerkannten Kirche (der athanasianischen wie der arianischen), die ja so autokratisch regierenden Kaisern wie Konstantin dem Gr., Constantius, Theodosius I. und Justinian als der Staatgewalt untergeordnet, als eine Staatsanstalt erschien, die Gegner auch rermittelst der Schule zu bekämpfen. So gebietet ein Edikt Liaiser Theodosius' II., daß man die Bücher des Nestorius verbrenne, daG niemand sich unterfangen solle etwas zu lernen, was nicht den Beschlüssen von Nicäa und Ephesus gemäß sei $^{{ }^{46}}$ ). Das Edikt bezog sich auf die Allgemeinheit, galt aber jedenfalls besonders für den Unterricht in der christlichen Lehre. Eine radikale Umwälzung in der Art des aus der Heidenzeit hergebrachten Schulbetriebes war freilich unmöglich ${ }^{47}$ ), sie erschien auch nicht nötig, solange die Schule sich dem Christentum gegenüber neutral oder wenigstens nicht aggressiv verhielt. Immer noch beruhte der Unterricht, abgesehen von den speziell theologischen Katechetenschulen, auf der Lektüre und Erklürung der heidnischen Klassiker, aber diese dienten

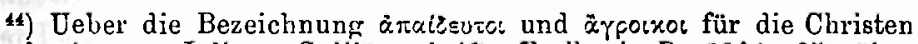
8. Rud. Asmus, Julians Galilïerschrift. Freib. i. B. 1904, 25, über Julians Ansicht, die christlichen Rhetoren soliten sich lieber der Erklürung der Bibel widmen, deren Studium freilich niemanden $\gamma \varepsilon w \alpha i o s$ mache, s. ibid. 24 f.; cf. 45.

45) Jeber ein angebliches Projekt des Gotenkönigs Totila, das die Vernichtung des Römertums durch Schlięung der rö̀mischen Schulen und Berufung germanischer Lehrer (!) bezweckt haben soll, spricht Jul. Ziehen nach Corrado Barbagallo, Lo stato et l'istruzione pubblica nell' impero Romano, Catanir 1911 (mir nicht zugänglich) in d. Berl. philol. Wochschr. 1911, 1296.

16) Mansi V 417. - Hertzberg a. 0. a. III 317.

17) Konstantin d. Gr. hielt die den Professoren und Aerzten von jeher gewührten Vorrechte, selbst die Befreiung vom Dekurionat aufrecht (Cod. Theod. XIII 3 ). 
nur als Mittel zu dem Zwecke, eine formale Bildung zu erlangen und die nötigen Kenntnisse und äußerliche Fertigkeiten sich anzueignen. In welchem Sinne der Christ sich mit der weltlichen Wissenschaft beschäftigen darf, darüber gibt einerseits

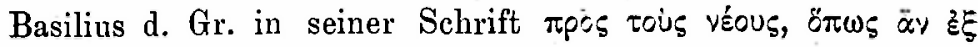

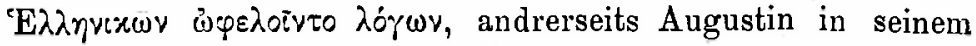
Werke De doctrina christiana besondere Anweisungen, nach denen man zwar das Nützliche aus den Werken der Heiden, besonders die artes liberales, anmehmen, aber dabei mit der größsten Vorsicht auf seine Seele achten soll ${ }^{48}$ ). Der Geist der Zeit wurde durch den Inhalt der heidnischen Schriftsteller nicht mehr berührt. An die Stelle Homers und Platos var das Evangelium getreten. Wie völlig die Schriften des Neuen wie Alten Testaments die Denktätigkeit der Menschheit aut sich gezogen und von der Gedankenwelt des klassischen Altertums abgelenkt hatten, das zeigt ein Blick in die Werke der großen Kirchenväter, eines Origenes und Johannes Chrysostomus wie eines Ambrosius und Augustinus, die doch ihre geistige Schulung und literarische Bildung den im Unterricht behandelten heidnischen Klassikern verdankten. Hieronymus ${ }^{49}$ ) fühlt Gewissensbedenken, wenn ihp̣n bei der Lektüre des Cicero zugerufen wird: "Ciceronianus es, non Christianus."

Wenn Libanius über die Unterdrückung der echten Bildung durch Kaiser Constantius klagt ${ }^{50}$ ), so bezieht sich dies nicht etwa auf ein Verbot der Lektüre heidnischer Autoren, sondern auf die Bevorzugung der römisch-juristischen Bildung vor der griechisch-sophistischen, welche durch die Notwendigkeit der Gewinnung zahlreicher, juristisch geschulter Beamten für den kaiserlichen Verwaltungsdienst bedingt war. Diesem Zwecke hatten auch vorzugsweise die kaiserlichen Hochschulen in Rom, Berytus und Konstantinopel zu dienen ${ }^{51}$ ), deren Ver-

$\left.{ }^{48}\right) \mathrm{Vgl}$. Hermann Eickhoff, Zwei Schriften des Basilius und des Augustinus, Schleswig 1897.

49) Ep. 22 ad Eustochium, c. 29. Cf. Gregor von Nazianz, ep. 235; Basilius, ep. 223.

so) Or. LXII p. 350,7 For.

51) Auch die Hochschule zu Athen war staatlich organisiert; vgl.

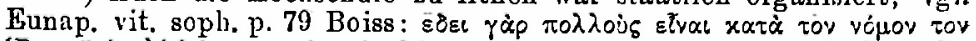

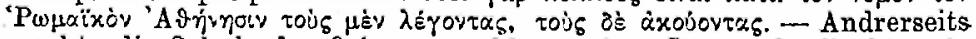
machte die Schule des Origenes wohl manchen Jünger des Rechts wie 
fassung im allgemeinen wohl derjenigen der Universität Kipel entsprach, die in einer Verordnung des Kaisers Theodosius II., der wie Konstantin d. Gr. ${ }^{52}$ ), Lehrern und Aerzten wiederum ihre Privilegien und Immunitäten bestätigte ${ }^{53}$ ), vom Jahre 425 im Codex Theodosianus ${ }^{54}$ ) vorliegt. In der Hauptsache wird dieselbe schon auf Konstantin d. Gr., der in der Nova Roma auch die Hochschule nach dem Muster der römischen gründete, zurückgehen ${ }^{55}$ ). Wie die Stellung und Lehrtätigkeit der Privatlehrer und insbesondere der Professoren ${ }^{56}$ ), so war auch der Studiengang, das Alter, die Führung der Studenten in dieser Zeit der Zentralisierung und Reglementierung durch die Vorschriften der Staatsregierung genau geregelt ${ }^{57}$ ). An die Stelle der freien Hingabe an die Wissenschaft war an dieser Einheitschule das erzwungene Brotstudium des zulünftigen Staatsdieners getreten, der Unterricht war vollständig rom Staate abhängig, der vor allem die Heranbildung gehorsamer und loyaler Beamter bezweckte, wie die Einleitung in Justinians Institutionen zeigt.

Die Aufsicht über die Schulen fällt dem Statthalter zu, wie besonders die Verhältnisse in Athen zeigen. Drastisch schildert Libanius ${ }^{58}$ ), wie sich die Lehrer um die Gunst der hohen Beamten bemübten, durch deren Einfluf sie auch rom

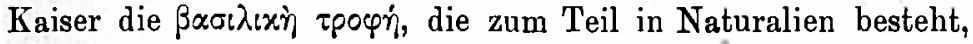

den späteren Bischof Gregorios Thaumaturgos von seinem Studium abtrünnig.

s2) Cod. Theod. XIII 3, 1.2. 3. - Gratian bestimmte in einem Gesetze vom J. 376 für Gallien (Cod. Theod. XIII 3,11) die Zahl der annonae für Grammatiker und Rhetoren.

ss) Cod. Theod. X III, 3, 16.

54) VI 21,1 , s. o. An. 32; XV 1, 53; cf. XIV, 9 (De studiis liberalibus urbis Romae et Constantinopolitanae).

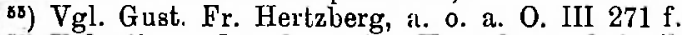

so) Valentinian I. nahm seine Verordnung betreff's der Lehrfreiheit (Cod. Theod. XIII 3,6 vom J. 364) bald wieder zuräck (Cod. Theod. XIII 3, 7 vom J. 369).

67) Vgl. die Verordnung der Kaiser Valentinian I., Valens und Gratian vom J. 370 im Cod. Theodos. XIV 9, 1 und das Prooemium der Institutionen Justinians.

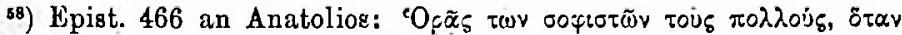

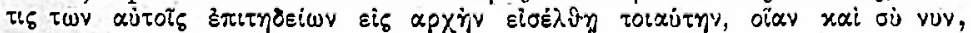

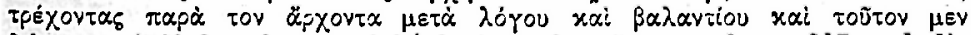

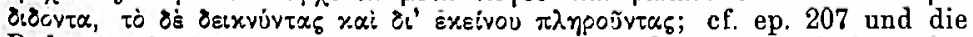

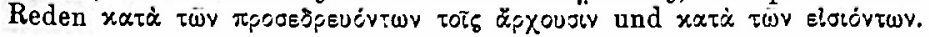




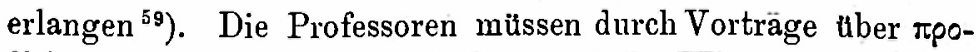

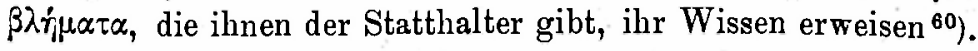
Bei den rhetorischen Wettkämpfen spielte der Prokonsul in Athen den Schiedsrichter. Säumte bei einer solchen Gelegenheit einer der Lehrer, so sandte er wohl auch Soldaten, um den, der der öffentlichen Probe im Theater fern bleiben wollte, zu holen ${ }^{61}$ ). Den Befehl eine Rede auf Kaiser Constans zu halten, erhält der berïhmte Sophist Prohäresius, der von Con-

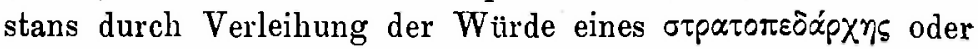
magister militum ausgezeichnet wurde ${ }^{62}$ ), rom praefectus praetorio ${ }^{63}$ ). Die Prokonsuln von Achaja traten, wie Hertzberg ${ }^{\text {(4) }}$ ) mit einem treffenden Vergleich bemerkt, "geradezu als Kuratoren der Universität Athen auf ${ }^{465}$ ).

Das Verhältnis von Schule und Staat wird schließlich von Justinian wie das zwischen Kirche und Staat im Sinne der Staatsomnipotenz geordnet. Die Rechtswissenschaft durfte seit 527 nur mehr an den eigentlichen Staatsuniversitäten zu Rom, Kpel und Berytus gelehrt werden. Jetzt wurde solchen, die „ am heidnischen Wahnsinn der unseligen Hellenen litten “, die Unterweisung der Jugend verboten ${ }^{66}$ ). Die christliche Regierung wollte mit den nämlichen Mitteln den Geist der Zeit beherrschen wie die heidnische Julians. In Jahre 529 endlich ward durch ein Edikt Justinians, das den Unterricht in der Philosophie verbot, die Universität Athen tatsächlich aufgehoben ${ }^{67}$ ), im nämlichen Jahre, in welchem der letzte heidnische

59) Liban, epist. 207. 488. - Themistins, or. 23 p. 291 Dind. Hertzberg a. o. a. O. III 318 - Cod. Theod. XIII, 3, 11.

o0) Fritz Schemmel, Die Hochschule von Athen, Neue Jahrb. XXII (1908) 494 fi. - Grasberger, Erziehung und Unterricht im klass. Altertum III 447. 453. Hertzberg a. o. a. O. III 85. 316. 319. Rauschen, Das griechisch-römische Schulwesen zur Zeit des ausgehenden antiken Heidentums, Bonn 1900, 21.

61) Eunap. vit. soph. p. 83 ff. Boiss.

6) Eunap. vit. soph. p. 79 Boiss.

o3) Eunap. vit. soph. p. 90 Boiss.

64) III 316.319 .

65) Wie derb der Prokonsul gegenüber den Gelehrten sein konnte, s. bei Eunap. vit. soph. 69 f. Boiss., wo er denselben erklärt: Ë $\sigma \in \sigma \vartheta \dot{\varepsilon} \gamma \varepsilon$

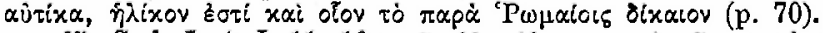

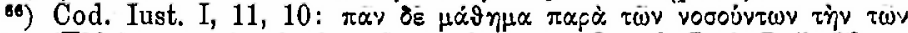

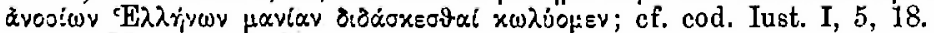

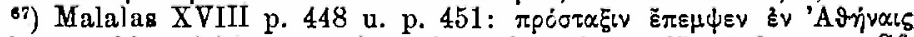

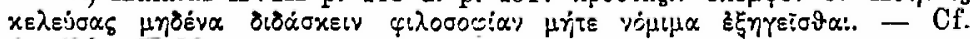
Agatbias II 30. 
Ueber d. Verhältnis von Staat u. Schule i. d. röm. Kaiserzeit. 191

Tempel in Italien, der des Apollo auf dem mons Casinus, vom Leiligen Benedikt in eine christliche Kirche verwandelt ward. Die freiere Hochschule war der Konkurrenz der rom Staate röllig abhängigen erlegen, ebenso wie die freiere theologische Forschung durch Unterdrückung der alten Schule des Origenes zu Gunsten der Staatskirche beseitigt worden war.

Mit der antiken Philosophie ward die antike Religion zu Grabe getragen. Die Reste des antiken Wissens aber wurden mit der Schule in die Kirche aufgenommen, deren Vertreter infolge der Passivität des Staates jetzt die Lehrer der Menschheit werden.

Nürnberg.

L. Hahn.

P.S.: Ueber des Libanius Kampf wider die Romanisierung der oriechischen Schule wird Verf. nächstens eine Abhandlung veröffentlichen. 